\title{
Correction to: Efficient and Secure Three-Factor User Authentication and Key Agreement Using Chaotic Maps
}

\author{
Jiaxi $\mathrm{Hu}$, Zhiqiang Xu, Debiao He, Sherali Zeadally, \\ and Kim-Kwang Raymond Choo
}

\section{Correction to:}

Chapter "Efficient and Secure Three-Factor User Authentication and Key Agreement Using Chaotic Maps" in: J. Vaidya et al. (Eds.): Cyberspace Safety and Security, LNCS 11982, https://doi.org/10.1007/978-3-030-37337-5_15

The original version of this chapter was revised. The affiliation of Sherali Zeadally was corrected to "College of Communication and Information, University of Kentucky, Lexington, USA." 